\title{
Are the late results of thymectomy satisfactory in the treatment of myasthenia gravis?
}

\author{
Berkant Özpolat
}

Department of Thoracic Surgery, Kırıkkale University, School of Medicine, Kırıkkale

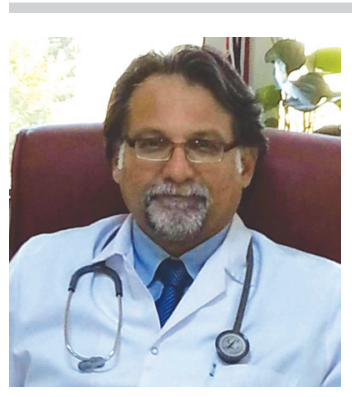

It is demonstrated that thymectomy is more effective than conservative treatment for myasthenia gravis (MG) regarding outcome, clinical improvement and remission rates in long-term follow-up $[1,2]$. The literature draw attention to patients with non-thymomatous MG obtaining significantly higher rates of complete stable remission than the patients with thymomatous MG [3-5]. The additional prognostic factors were stated as steroid therapy, age of onset, short duration of the disease and finally positive acetylcholine receptor antibodies [1,4-6].

The results of a recent survey of current surgical practice in thymic disease amongst EACTS members showed that, $80.8 \%$ of the surgeons perform thymectomy for both MG and thymic cancer, $78.4 \%$ have a strict cooperation with dedicated neurologist and anesthesiologist during the course of treatment and 55.8\% operate thymic hyperplasia with Stage I MG (ocular myasthenia) [7].

Although there is no published information, in most of the clinics that I communicate during my surgical practice there has been a debate between neurologists and thoracic surgeons if surgery should be a part of MG treatment. Unfortunately the resistance of some neurologists to surgery for suitable patients was difficult to understand which might be due to obstacles to establish a multidisciplinary team. Under these circumstances it is gratifying to see the results of comparison of the 1st and 5th year outcomes of thymectomy by Dural et al. in this issue of the Turkish Journal of Clinics and Laboratory. The late results of 27 patients underwent thymectomy are consistent with the literature i.e. no significant difference were found in the late outcomes. They concluded that thymectomy is a safe and reliable method for the treatment of MG.

Currently extended transsternal thymectomy remains as the gold standard tool to achieve complete remission rates in MG [1,3-6]. Late results demonstrated that complete remission rates were significantly better in extended transsternal thymectomies compared to basic transsternal thymectomies [8]. Recent studies show that minimally invasive surgery was found to be superior in terms of improvement in MG -associated symptoms. Additionally, the hospital stay was shorter, and the patients felt less disturbed by direct effects of the operation. Therefore, minimally invasive thymectomy can also be a treatment of choice for patients undergoing surgery for MG $[9,10]$. Although the evidence is low, whatever technique is used the main stay is to leave less thymic tissue behind for better long term results [6].

As a take home massage after evaluating those results, extended thymectomy should be recommended in patients with early onset, generalized MG and positive acetylcholine receptor antibodies, and associated thymoma.

\section{References}

1. Huang CS, Hsu HS, Huang BS, Lee HC, Kao KP, Hsu WH, Huang MH. Factors influencing the outcome of transsternal thymectomy for myasthenia gravis. Acta Neurol Scand 2005; 112: 108-14.

2. Bachmann K, Burkhardt D, Schreiter I, et al. Thymectomy is more effective than conservative treatment for myasthenia gravis regarding outcome and clinical improvement. Surgery 2009; 145: 392-8.

3. Yu S, Li F, Chen B, Lin J, Yang M, Fu X, Li J, Bu B. Eight-year follow-up of patients with myasthenia gravis after thymectomy. Acta Neurol Scand 2015; 131:94-101.

4. Park IK, Choi SS, Lee JG, Kim DJ, Chung KY. Complete stable remission after extended transsternal thymectomy in myasthenia gravis. Eur J Cardiothorac Surg 2006; 30: 525-8.

5. Masaoka A, Yamakawa Y, Niwa H, Fukai I, Kondo S, Kobayashi M, Fujii Y, Monden Y. Extended thymectomy for myasthenia gravis patients: a 20-year review. Ann Thorac Surg. 1996; 62: 853-9.

6. Van Schil PE, Mercelis R, Lucchi M. Extended versus standard thymectomy for myasthenia gravis. In MK Ferguson (ed.). Difficult Decisions in Thoracic Surgery. An Evidence-based approach 1. Springer-Verlag London. 2014. p. 677-687.

7. Lucchi M, Van Schil P, Schmid R, Rea F, Melfi F, Athanassiadi K, Zielinski M, Treasure T; EACTS Thymic Working Group. Thymectomy for thymoma and myasthenia gravis. A survey of current surgical practice in thymic disease amongst EACTS members. Interact Cardiovasc Thorac Surg 2012; 14: 765-770.

8. Zieliński M, Kuzdzal J, Szlubowski A, Soja J. Comparison of late results of basic transsternal and extended transsternal thymectomies in the treatment of myasthenia gravis. Ann Thorac Surg 2004; 78: 253-8.

9. Roth T, Ackermann R, Stein R, Inderbitzi R, Rösler K, Schmid RA. Thirteen years follow-up after radical transsternal thymectomy for myasthenia gravis. Do short-term results predict long-term outcome? Eur J Cardiothorac Surg. 2002; 21: 664-70.

10. Bachmann K, Burkhardt D, Schreiter I, et al. Long-term outcome and quality of life after open and thoracoscopic thymectomy for myasthenia gravis: analysis of 131 patients. Surg Endosc 2008; 22: 2470-7. 